\title{
Effect of pH on the Binding of $\beta$-Lactoglobulin to Sodium Polystyrenesulfonate
}

\author{
R. K. Hallberg and P. L. Dubin* \\ Department of Chemistry, Indiana-Purdue University, 402 North Blackford Street, \\ Indianapolis, Indiana 46202-3274
}

Received: June 23, 1998

\begin{abstract}
The binding of $\beta$-lactoglobulin to the synthetic polyanion, sodium polystyrenesulfonate, was studied by frontal analysis continuous capillary electrophoresis (Gao et al. Anal. Chem. 1997, 69, 2945). The data were fit to a modified Scatchard plot, and the intrinsic binding constant, $K_{\mathrm{obs}}$, was measured as a function of $\mathrm{pH}$ at fixed ionic strength of $0.05 \mathrm{M}$. The $\mathrm{pH}$ dependence of $K_{\mathrm{obs}}$ was found to follow the semi-logarithmic dependence of $K_{\text {obs }}$ on protein charge $Z$ predicted by Lohman and Record, despite the fact that the net protein charge was of the same sign as the polyanion. However, the magnitude of $\partial \log K_{\mathrm{obs}} / \partial Z$ did not agree with the predicted value, either for this system or for pentalysine/DNA data. The current results suggest that the free energy of binding of a protein to a synthetic polyelectrolyte depends on some local protein charge that may vary linearly with the net protein charge.
\end{abstract}

\section{Introduction}

Proteins interact strongly with both natural polyelectrolytes and synthetic polyelectrolytes. With regard to the former, studies have largely been focused on the interactions of nucleic acids and proteins in the transcription process, ${ }^{1-6}$ although there is growing interest in the association of proteins with other biological polyelectrolytes such as heparin or hyaluronic acid. ${ }^{7}$ On the other hand, studies of the interaction of proteins with synthetic polyelectrolytes have been considered in the context of a wide array of applications including drug delivery, ${ }^{8,9}$ enzyme activation and stabilization, ${ }^{10,11}$ protein separation, ${ }^{12-15}$ and protein purification. ${ }^{16,17}$ Despite the fundamental similarity of these two types of efforts, they are usually studied from the perspectives of different disciplines and are quite effectively segregated in the literature. In particular, it could appear that the specificity and complementary design of proteins and polyelectrolytes that interact in the biological milieu might have no counterpart in artificial mixtures. However, because the compositions of synthetic polyelectrolytes-and hence their hydrophobicity, charge density, and chain stiffness-can be altered more systematically than those of natural polyelectrolytes, resolution of various contributions to the interaction may be feasible in a way not possible for purely natural systems. Thus, elucidation of the behavior of quasi-synthetic systems may facilitate better understanding of the natural systems.

A wide variety of hydrodynamic, chromatographic, and spectroscopic techniques can be used to study polyelectrolyteprotein complexes (for a review, see ref 18). For systems involving flexible chain "random coil" (e.g., synthetic) polyelectrolytes, many of these studies focus on the question of the long-range structure and conformation of intrapolymer and/or multipolymer complexes. However, for complexes involving DNA, the unique structure of this polyelectrolyte alters the nature of such questions. These distinctions do not apply to thermodynamic characterization, and thus the intrinsic binding constant is a key parameter for both natural and partly synthetic protein-polyelectrolyte systems.

A priori, the intrinsic binding constant is expected to be predominantly electrostatic and therefore dependent on variables

\footnotetext{
$\dagger$ Present address: Dow AgroSciences LLC, 9330 Zionsville Road,
} Indianapolis, IN 46268. such as the total protein charge $(Z)$ (and hence on the $\mathrm{pH}$ of the system), the ionic strength (I); and the charge density of the polyelectrolyte. Much of the motivation for understanding the physical chemistry underlying the influence of $Z$ and $I$ on $K_{\mathrm{b}}$ arises from interest in protein-DNA interactions. For example, Record and co-workers ${ }^{19}$ analyzed binding data for DNA and oligopeptides in terms of the McGhee and von Hippel ${ }^{20}$ site binding model in which a ligand of charge $z_{2}$ replaces some number of DNA counterions of charge $z_{1}$. The thermodynamic treatment of Record et al., based on Manning's counterion condensation theory, ${ }^{21}$ leads to the result that the measured binding constant, $K_{\mathrm{obs}}$, defined in terms of the concentration of DNA(D) and all ligand species (L):

$$
K_{\mathrm{obs}}=[\mathrm{LD}] /[\mathrm{L}][\mathrm{D}]
$$

varies with the concentration of the salt raised to the $-z_{2} / z_{1}$ power. Using a similar approach, Record et al. obtained a relationship at constant ionic strength between $K_{\text {obs }}$ and ligand charge $Z::^{22}$

$$
\log K_{\mathrm{obs}}=\log K^{0}-Z \psi \log \left[\mathrm{M}^{+}\right]
$$

Where $K^{0}$ is the equilibrium constant for the reaction

$$
\mathrm{L}+\mathrm{D}=\mathrm{L}-\mathrm{D}+\mathrm{Z} \psi \mathrm{M}^{+}
$$

where $\psi$ is the fraction of a counterion $\left(\mathrm{M}^{+}\right)$"thermodynamically associated" with the DNA (per phosphate unit) and [M+] is the added monovalent salt. This relationship was demonstrated for inter alia the model system of pentalysine and DNA acid. The validity of eq 2 for partly synthetic systems is the subject of the present study.

The above-mentioned treatments are based on counterion condensation theory; they take into account neither the structural characteristics of the polyelectrolyte nor the size and charge distribution of the ligand. The last issue is particularly significant for proteins that indeed are known to bind to polyelectrolytes on the wrong side of their isoelectric points. ${ }^{23}$ Rouzina and Bloomfield ${ }^{24}$ approached these issues in the context of DNA-protein interactions. By exact solution of the Pois- 
son-Boltzmann equation, they obtained analytical expressions for the amount of bound ligand and its dependence on $z_{2}$ and $z_{1}$ and DNA charge density. ${ }^{24 a}$ They extended the treatment to large ligands with charge distributions, essentially by deducing an effective charge value for $z_{2}$ from geometric considerations. ${ }^{24 b}$

The foregoing treatments focus on the interactions of DNA with ligands that are viewed as multivalent cations and contain as a central theme the release of a stoichiometric number of counterions from DNA upon ligand binding. In these models, the polyelectrolyte is a rigid, uniformly charged cylinder, and the ligand is treated as a virtual point charge, the result being the dependence of the binding constant only on the ligand valence and on the counterion concentration. However, in general, electrostatic interactions in colloidal systems are not stoichiometric. $^{25}$ An entirely different approach to polyelectrolyte-protein binding may be derived from treatments for the adsorption of flexible chain polyelectrolytes onto oppositely charged surfaces. ${ }^{26-28}$ In this case, we view the protein as a surface onto which some set of contiguous segments may adsorb. The role of the small ion is then viewed in terms of its screening and its influence on the polyelectrolyte dimensions, as opposed to a stoichiometric competition with the protein for the polyelectrolyte. Entropic effects are assigned to changes in polyelectrolyte configuration and not primarily to small ion release. The consequences of this analysis are also quite different; namely, polyelectrolyte adsorption appears as a phase transition, occurring only when the ionic strength falls below the value of $\kappa$ given by an equation of the form

$$
\xi \sigma / \kappa^{a}=\text { constant }
$$

in which $\kappa$ is the Debye-Hückel parameter, $\sigma$ is the colloid surface charge density (in this case the protein surface charge density), and $\xi$ is the charge per polymer repeat unit, i.e., proportional to the polymer linear charge density. While the polyelectrolyte's partner in this scenario was a planar surface in the early treatments, the introduction of surface curvature apparently retains much of the important features of eq $3 . .^{29,30}$ A well defined point of incipient complex formation corresponding to the physical meaning of eq 3 has indeed been observed for flexible chain polyelectrolytes interacting with $5-20-\mathrm{nm}$ colloidal (micellar) particles. ${ }^{31}$ Similar results have

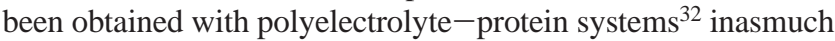
as the $\mathrm{pH}$ at which complexes first appear corresponds to a critical net protein charge $Z$ which varies with the square root of ionic strength; ${ }^{33}$ although it is noteworthy that no discontinuity in this relationship is observed at $Z=0$ with both cationic and anionic polyelectrolytes showing binding to bovine serum albumin on the wrong side of the isoelectric point. These results demonstrate a direct dependence of critical protein surface charge density on $\kappa$ as predicted by eq 3 with $a=1$.

The system under study in the present work partakes of some of the features of both sets of approaches described above. The polyelectrolyte sodium polystyrenesulfonate (NaPSS) is a flexible chain, not a rigid cylinder like DNA, and thus corresponds to the second set of theoretical models. ${ }^{26-30}$ On the other hand, the "ligand" $\beta$-lactoglobulin may be viewed as intermediate between the planar surfaces described in refs $26-30$ and the effective point charge envisaged in refs 19 and $21-23$. The interaction is expected to be predominantly electrostatic with the binding dictated by the ionic strength and the $\mathrm{pH}$, which controls the protein charge. Specifically, the goal of this work is to test the validity of the model embodied in eq 2 and eq 3 for this system.

The binding of $\beta$-lactoglobulin to NaPSS was measured using frontal analysis continuous capillary electrophoresis (FACCE), ${ }^{34}$ a modified version of frontal analysis capillary electrophoresis. This technique facilitates the acquisition of protein-polyelectrolyte binding data in a rapid and reproducible manner that compares favorably with classical methods such as membrane equilibrium, or equilibrium ultracentrifugation. Sample injection and separation take place concurrently in FACCE, so there are no sample injection parameters to consider. Electropherograms display continuous plateaus rather than distinct peaks, with unbound protein eluting first. ${ }^{34 a}$ Quantitation of this free protein can be done over a suitable range of concentrations and without concern about perturbation of the binding equilibrium. Analysis of the binding curves can yield information about the binding constant, the size of the binding site (degree of polymerization of the polyelectrolyte relative to the number of proteins bound per polymer chain at saturation), and the degree of cooperativity.

\section{Experimental Section}

Reagents and Solutions. $\beta$-Lactoglobulin A\&B (catalog no. L-2506) was purchased from Sigma Chemical Co. (St. Louis, MO). Sodium poly(styrenesulfonate) (NaPSS) of $M_{\mathrm{w}}=710000$ with $M_{\mathrm{w}} / M_{\mathrm{n}}<1.1$ was obtained from Pressure Chemical Co. (Milwaukee, WI). A second sample of NaPSS was prepared by polymerization of sodium styrenesulfonic acid in pure water with ammonium persulfate as initiator, followed by dialysis and freeze-drying. From the measured diffusion coefficient, the $M_{\mathrm{W}}$ was estimated as $2.5 \times 10^{5}$. Buffers were prepared at the desired $\mathrm{pH}$ with a constant ionic strength of $0.05 \mathrm{M}$ using monobasic and dibasic sodium phosphate purchased from Fisher Scientific Co. and Milli-Q water. Stock solutions of NaPSS at approximately five times the final concentration $(1 \mathrm{mg} / \mathrm{mL})$ were prepared in the appropriate buffer, sonicated in a bath for approximately $15 \mathrm{~min}$, and allowed to stand for at least $1 \mathrm{~h}$. Stock solutions of $\beta$-lactoglobulin were prepared using the appropriate buffer in the approximate desired concentration (2-3 $\mathrm{mg} / \mathrm{mL}$ ) and diluted to provide a series of different concentrations. Accurate concentrations for each solution were determined via UV spectrometry using an extinction coefficient of $\epsilon=18.6 \mathrm{dL} / \mathrm{g} \mathrm{cm}$ for NaPSS at $262 \mathrm{~nm},{ }^{35}$ and an absorbance of 9.5 at $280 \mathrm{~nm}$ for a $1 \%$ solution of $\beta$-lactoglobulin. ${ }^{36}$ Mixtures of NaPSS and $\beta$-lactoglobulin were prepared by dividing each member of the series of $\beta$-lactoglobulin solutions into two equal parts and diluting one 4:1 $\beta$-lactoglobulin:NaPSS solution and the other 4:1 $\beta$-lactoglobulin:buffer. The NaPSSfree solutions were used to prepare the calibration curve.

Apparatus and Operating Conditions. UV spectra were measured with a Beckman (Fullerton, CA) DU-70 spectrophotometer and a matched set of $1-\mathrm{cm}$ quartz cells (Fisher). Capillary electrophoresis was performed using a Beckman (Fullerton, CA) P/ACE $5500 \mathrm{CE}$ with a photodiode array detector, operating at $7 \mathrm{kV}$ and $25^{\circ} \mathrm{C}$. The fused silica capillary (Polymicro Technologies Inc., Phoenix, AZ) of dimensions 50 $\mu \mathrm{m} \times 27 \mathrm{~cm}$ (20 cm from injection to detection window) was prepared prior to each set of experiments by washing with 0.1 $\mathrm{N}$ sodium hydroxide $(\mathrm{NaOH})$ for $10 \mathrm{~min}$ followed by a 5-min wash with water. A 1-min wash with $\mathrm{NaOH}$ followed by a 2-min rinse with buffer was performed prior to each individual run.

The FACCE experiment is initialized by equilibrating the capillary with buffer for $2 \mathrm{~min}$. The inlet end of the capillary is then placed in a vial containing the equilibrated sample solution, and the outlet end is placed into a vial containing buffer. Constant voltage is applied, and separation, manifested 


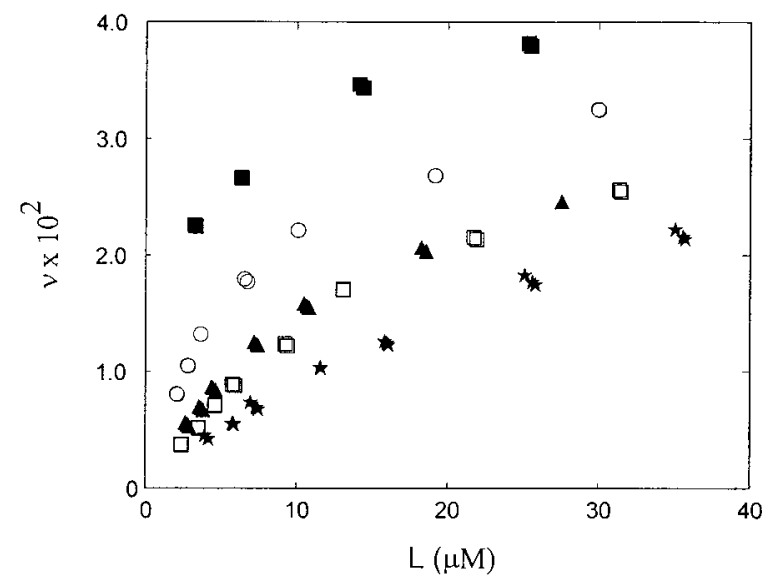

Figure 1. Binding isotherms for $\beta$-lactoglobulin and sodium polystyrenesulfonate at various $\mathrm{pH}$ values $(I=0.05 \mathrm{M})$ : (回) 6.27 , (O) 6.55 , (৯) $6.70,(\square) 6.85,(\star) 7.00$.

in continuous plateaus, is observed. The first eluting plateau is the unbound protein, and the second is the proteinpolyelectrolyte complex. The concentration of unbound protein is determined using the height of the first plateau, and the calibration curve is constructed by measuring the plateau height of known concentrations of protein using the same experimental conditions as for the protein-polyelectrolyte mixture. The extent of binding $(v)$ is determined from the amounts of unbound and total protein.

\section{Results and Discussion}

Conditions for measurements of binding isotherms were established by first determining the range of $\mathrm{pH}$ values corresponding to soluble complex formation, using turbidimetry. ${ }^{32}$ The point of phase separation (coacervation) for a mixture of $0.2 \mathrm{~g} / \mathrm{L} \beta$-lactoglobulin and $0.2 \mathrm{~g} / \mathrm{L}$ NaPSS was established as $\mathrm{pH}_{\phi}=5.2$ at $I=0.05 \mathrm{M}$. Turbidimetry also indicated that the onset of protein-polyelectrolyte complexation occurred at $\mathrm{pH}_{\mathrm{c}}=8.5$. FACCE measurements revealed that the binding below $\mathrm{pH} 6.27$ was too strong to allow for any detectable free protein at low protein concentrations, while the binding diminished drastically above $\mathrm{pH}$ 7. Thus, while soluble complexes may exist between $\mathrm{pH} 8.5$ and $\mathrm{pH} 5.2$, the range of $\mathrm{pH}$ values studied here was more limited. Binding curves were obtained by varying the protein concentration at fixed polymer content in the $\mathrm{pH}$ range from 6.2 to 7 , with the results shown in Figure 1. Multiple measurements indicate the excellent reproducibility of this method.

NaPSS obtained from Pressure Chemical Company is known to have varying degrees of sulfonation, as low as ca. $80-85 \% .{ }^{35}$ Such a low degree of sulfonation could lead to hydrophobic contributions to binding. For this reason, a second sample of NaPSS was prepared by free radical polymerization of sodium styrenesulfonate to assure $100 \%$ sulfonation. The binding isotherm obtained with this material at $\mathrm{pH} 6.47$ was essentially identical to that of the Pressure Chemical sample.

In order to extract the intrinsic binding constants $\left(K_{\mathrm{obs}}\right)$, the data were fitted to the equation proposed by McGhee and von Hippel: ${ }^{29}$

$$
v / L=K_{\mathrm{obs}}(1-n v)((1-n v) /(1-(n-1) v))^{n-1}
$$

where $v$ is the number of protein molecules bound per polyelectrolyte residue, $L$ is the concentration of unbound protein, and $n$ is the number of polyelectrolyte residues per binding site. Fitting of the data to eq 4 was accomplished using

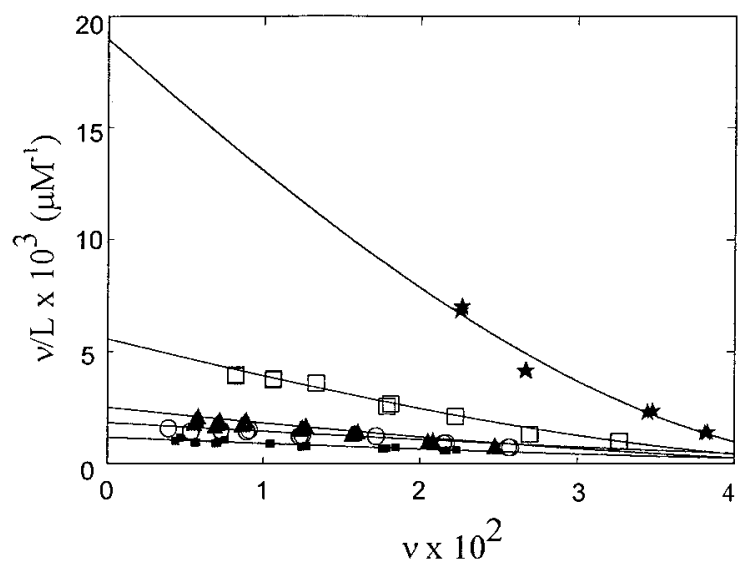

Figure 2. Scatchard plots for the binding of $\beta$-lactoglobulin to sodium polystyrenesulfonate at various $\mathrm{pH}$ values $(I=0.05 \mathrm{M})$ : (回) 6.27,

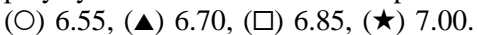

TABLE 1: Binding Constant and Binding Site Size at Various pH Values in $0.05 \mathrm{M}$ Buffers

\begin{tabular}{crcc}
\hline $\mathrm{pH}$ & $-Z^{a}$ & $\log K_{\text {obs }}\left(\mathrm{M}^{-1}\right)^{b}$ & $n^{b}$ \\
\hline 6.27 & 9.06 & $4.28 \pm 0.03$ & $16.8 \pm 0.5$ \\
6.55 & 10.64 & $3.75 \pm 0.01$ & $15.9 \pm 0.5$ \\
6.70 & 11.61 & $3.40 \pm 0.01$ & $14.9 \pm 0.6$ \\
6.85 & 12.45 & $3.26 \pm 0.01$ & $11.6 \pm 0.5$ \\
7.00 & 13.25 & $3.07 \pm 0.01$ & $12.5 \pm 0.8$
\end{tabular}
4

${ }^{a}$ By interpolation from ref $38 .{ }^{b}$ By fitting experimental data to eq

the JMP IN software (SAS Institute Inc.), which provided the best fit values of $K_{\mathrm{obs}}$ and $n$ along with the standard deviation for these parameters. The "modified Scatchard" plots corresponding to eq 4 are shown in Figure 2. Scatter at low $v$ values is more evident in this plot; these deviations result from measurements at low concentrations where the error of $0.1 \mathrm{mAU}$ is significant relative to a signal on the order of $2 \mathrm{mAU}$. According to McGhee and von Hippel, ${ }^{20}$ the convex shape of the isotherms may be consistent with the binding of a non-selfinteracting ligand that is able to overlap more than one lattice site. Values of $K_{\text {obs }}$ and $n$ are summarized in Table 1 , along with the net charge on $\beta$-lactoglobulin, interpolated from literature $\mathrm{pH}$ titration curves. ${ }^{37}$ According to eq 2, the dependence of $K_{\mathrm{obs}}$ on $\mathrm{pH}$ at constant ionic strength is given by $K_{\text {obs }}=K^{0}\left[\mathrm{M}^{+}\right] \psi^{Z}$, where $\left[\mathrm{M}^{+}\right]=0.05 \mathrm{M}, \psi$ is the fraction of $\mathrm{Na}^{+}$"thermodynamically bound" to NaPSS, and $Z$ is the ligand charge. The linearity of the plot of $\log K_{\mathrm{obs}}$ vs $\log Z$ in Figure 3 (regression coefficient $r^{2}=0.98$ ) is consistent with eq 2 , as is the positive slope, which should be equal to $-\psi \log \left[\mathrm{M}^{+}\right]$according to eq 2 . However, the slope of Figure 3 is found to be 0.29 , which is in poor agreement with the value of $-\psi \log \left[\mathrm{M}^{+}\right]$of 1.08 calculated using $\psi=0.83$ for NaPSS. ${ }^{34 \mathrm{~b}}$ Furthermore, we must remember that the binding measurements are all made under $\mathrm{pH}$ conditions where the net protein charge is of the same sign as that of the polyelectrolyte, which would seem to invalidate the fundamental approach of refs $20-22$ that underlie eq 2.

One explanation for binding on the wrong side of the isoelectric point might invoke a hydrophobic interaction between NaPSS and the protein, introducing a non-electrostatic effect that overcomes repulsion. However, we note that sodium polyvinylsulfate also binds bovine serum albumin, lysozyme, and ribonuclease on the wrong side of their isoelectric points..$^{23}$ Certainly in these cases it is difficult to invoke hydrophobic interactions to explain the "anomalous" binding, since the bulky sulfonate groups of the polyanion are easily seen by molecular 


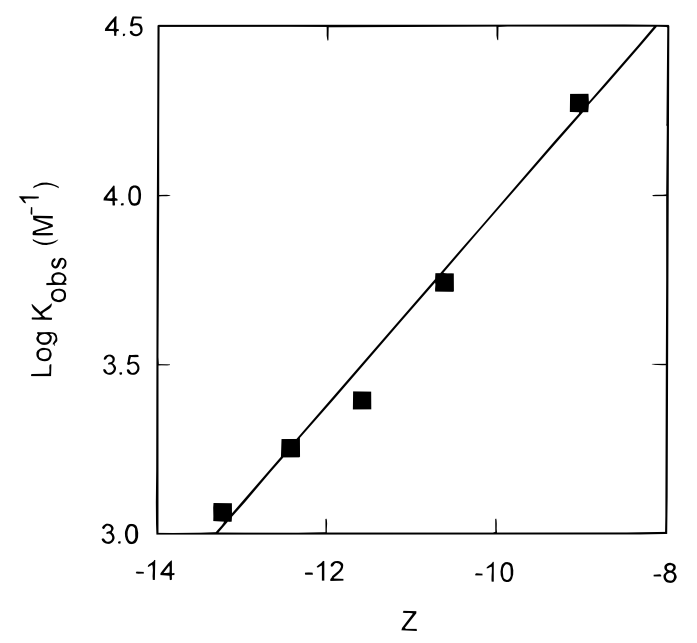

Figure 3. Dependence of the observed binding constant for $\beta$-lactoglobulin and sodium polystyrenesulfonate on the total protein charge at $I=0.05 \mathrm{M}$.

TABLE 2: Binding of Pentalysine to DNA in 0.191 M $\mathrm{NaCl}^{22}$

\begin{tabular}{lcc}
\hline $\mathrm{pH}$ & $\mathrm{Z}$ & $\log K_{\text {obs }}\left(\mathrm{M}^{-1}\right)$ \\
\hline 6.05 & 4.88 & 2.9 \\
6.83 & 4.54 & 2.6 \\
7.63 & 4.15 & 2.1 \\
8.4 & 3.96 & 1.9 \\
8.81 & 3.84 & 1.8
\end{tabular}

modeling to preclude protein access to the hydrophobic polymer backbone. Secondly, the ionic strength dependence of the binding of $\beta$-lactoglobulin to NaPSS conforms to a linear dependence of $\log K_{\mathrm{obs}}$ on $\log I,{ }^{38}$ which is consistent with the ionic strength dependence predicted by purely electrostatic considerations. ${ }^{19}$ Were there to be a significant hydrophobic contribution, $K_{\phi}$, the observed linear $\log -\log$ dependence could only arise if $K_{\phi}$ had the same ionic strength dependence as the electrostatically driven binding constant, an unlikely result.

These inconsistencies are better rationalized by hypothesizing that the interaction of the protein with polyelectrolyte is controlled by an effective local protein charge, $z_{\text {eff }}$, smaller than $Z$ and opposite to it in sign. Such a "charge patch" has been previously proposed to explain binding of polyelectrolytes to proteins of the same sign. ${ }^{32,33}$ The linearity between $\log K_{\text {obs }}$ and $Z$ from eq 2 could be preserved if $z_{\text {eff }}$ varies linearly with $Z$. Accordingly, we would modify eq 1 and write:

$$
\log K_{\mathrm{obs}}=\log K^{0}-(f Z) \psi \log \left[\mathrm{M}^{+}\right]
$$

where $f$ represents the relationship between "patch charge" and net charge as $f=\left|z_{\text {eff }} / Z\right|$. This hypothesis derives support from previous turbidity and light scattering results, which showed that the critical conditions for protein-polyelectrolyte complex formation could be represented by linear plots of $Z_{\text {crit }}$ vs $\kappa$, which encompassed both negative and positive values of $Z_{\text {crit }}$.

It is also appropriate to consider the validity of eq 2 using the more suitable experimental situation of pentalysine bound to DNA $^{22}$ at an ionic strength of 0.19 M. Data interpolated from ref 22 are shown in Table 2 and plotted in Figure 4 to give a straight line with a regression coefficient of $r^{2}=0.996$. However, based on $\psi=0.88$ for DNA, ${ }^{22}$ the predicted slope is 0.63 , in rather poor agreement with the measured slope of 1.1 .

Observations from these analyses may be summarized as follows. The experimental results confirm that for two different

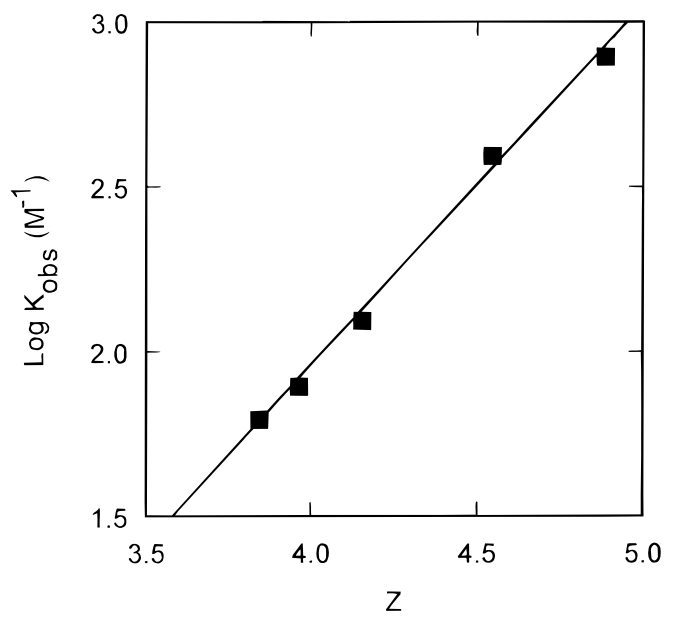

Figure 4. Dependence of the observed binding constant for pentalysine and T7 DNA on the total protein charge at $I=0.191 \mathrm{M}$. Data extrapolated from Figure 5 in ref 22.

but related systems - a flexible polyelectrolyte binding a protein and DNA binding oligolysine- $-\log K_{\mathrm{obs}}$ varies in a linear manner with the ligand charge at fixed ionic strength. On the one hand, these results might be considered as strong confirmation of the treatment of ref 22, even for the variant system of protein with flexible chain polyelectrolyte. On the other hand, the poor agreement of $\partial \log K_{\text {obs }} / \partial Z$ with the calculated values in both cases suggests the possibility of alternative interpretations of the data. Indeed, the linearity of Figures 3 and 4 essentially indicate that the free energy of binding is a linear function of ligand charge, and models other than those based on stoichiometric exchange might lead to a similar result.

The fitted values for $n$ in Table 1 show an increase in the size of the binding site (number of polyelectrolyte residues per bound protein) by almost $50 \%$ as the $\mathrm{pH}$ is decreased from 7.00 to 6.27 , an effect well above the error in estimating $n$. Thus, the number of proteins bound per polymer molecule decreases while the binding constant increases. We attribute this effect to an increase in the number of polymer residues that interact with a given protein. At lower $\mathrm{pH}$, the site of binding on a protein molecule, which is likely to correspond to a local region of net positive charge, is large enough to accommodate a polymer segment comprising on the order of 17 contiguous residues (contour length ca. $5 \mathrm{~nm}$ ). However, at higher $\mathrm{pH}$ the dimensions of such a positive patch on the protein must diminish, and then can only accommodate a polymer segment of about 12 residues that bind cooperatively (contour length ca. $3.5 \mathrm{~nm}$ ). This decrease in the size of the binding site results in a decrease in the binding constant accompanied by an increase in the number of proteins bound per polymer chain at saturation.

Last, we discuss the question of phase-transition type behavior at $\mathrm{pH}_{\mathrm{c}}$. The binding constant for $\beta$-lactoglobulin to NaPSS changes by a factor of 16 over a $\mathrm{pH}$ range of $0.7 \mathrm{pH}$ unit. This is considerably more abrupt than the binding constant for pentalysine to DNA, which changes by an order of magnitude when the $\mathrm{pH}$ changes by nearly $3 \mathrm{pH}$ units. If the binding process for the former involves the rearrangement of a number of contiguous polyelectrolyte repeat units, moving in concert, some cooperative characteristic could be imparted to the initial binding step. This could give rise to the large $\partial K_{\mathrm{obs}} / \partial \mathrm{pH}$ and the concomitant rather abrupt turbidimetric changes at a critical $\mathrm{pH}$ corresponding to the phase-transition-like behavior previously noted. ${ }^{32}$ 


\section{Conclusions}

The binding of $\beta$-lactoglobulin to a synthetic polyelectrolyte, polystyrenesulfonate, displays a $\mathrm{pH}$ dependence consistent with the observations and model presented by Record and co-workers for the electrostatic binding of cationic ligands to DNA, in that the binding constant varies in an exponential manner with the protein (ligand) charge $Z$ at fixed ionic strength. However, at the ionic strength employed here, binding is observed when the protein bears a negative net charge, i.e., of like sign to the polyelectrolyte. These results may point to a modification of the model of Record et al. wherein the interaction is dictated by a local or effective protein charge that is opposite in sign to the global charge but nevertheless depends on it in a linear way. On the other hand, it may be appropriate to consider an alternate model, leading to a binding free energy that is linear with $Z$, without solely invoking a stoichiometric displacement of counterions.

Acknowledgment. This work was supported by National Science Foundation Grants DMR-9619772 and CHE-9505953.

\section{References and Notes}

(1) Shaner, S. L.; Melancon, P.; Lee, K. S.; Burgess, R. R.; Record,

M. T., Jr. Cold Spring Harbor Symp. Quant. Biol. 1983, 47, 463.

(2) Latt, S.; Sober, H. Biochemistry 1967, 6, 3293. $26,212$.

(3) Poliakow, M.; Champagne, M.; Daune, M. Eur. J. Biochem. 1972,

(4) Yamamoto, K.; Alberts, B. J. Biol. Chem. 1974, 249, 7076.

(5) Jensen, D.; von Hippel, P. J. Biol. Chem. 1976, 251, 7198.

(6) Daune, M. Eur. J. Biochem. 1972, 26, 207.

(7) Riggs, A.; Suzuki, H.; Bourgeois, S. J. Mol. Biol. 1970, 53, 401.

(8) Regelson, W. Interferon 1970, 6, 353. 160.

(9) Ottenbrite, R. M.; Kaplan, M. M. Ann. N.Y. Acad. Sci. 1985, 446,

(10) Margolin, A.; Sheralijuk, S. F.; Izumrudov, V. A.; Zezin, A. B.; Kabanov, V. A. Eur. J. Biochem. 1985, 146, 625.

(11) Burgess, R. R.; Jendrisak, J. J. Biochemistry 1975, 14, 4634.

(12) Wang, Y.; Gao, J. Y.; Dubin, P. L. Biotechnol. Prog. 1996, 12,
(13) Cifuentes, A.; Poppe, H.; Kraak, J. C.; Erim, F. B. J. Chromatogr.

$B$ 1996, 681, 21 .

(14) Sternberg, M. Process Biochem. 1976, 11, 11.

(15) Berdick, M.; Morawetz, H. J. Phys. Chem. 1953, 57, 959.

(16) Shieh, J.; Glatz, C. E. Am. Chem. Soc., Div. Polym. Chem. Prepr. 1991, 32 (1), 606.

(17) Strege, M. A.; Dubin, P. L.; West, J. S.; Daniel Flenta, C. D. Protein Purification: From Molecular Mechanism to Large-scale Process; American Chemical Society: Washington, DC, 1990, Chapter 5.

(18) Xia, J.; Dubin, P. In Macromolecular Complexes in Chemistry and Biology; Dubin, P. L., Bock, J., Davis, R. M., Schulz, D., Thies, C., Eds.; Springer-Verlag: Berlin Heidelberg, 1994; Chapter 15.

(19) Record, M. T., Jr.; Anderson, C. F.; Lohman, T. M. Q. Rev. Biophys. 1980, 11, 103 .

(20) McGhee, J. D.; von Hippel, P. H. J. Mol. Biol. 1974, 86, 469.

(21) Manning, G. S. Q. Rev. Biophys. 1978, 11, 179.

(22) Lohman, T. M.; deHaseth, P. L.; Record, M. T., Jr. Biochemistry 1980, 19, 3522.

(23) Park, J. M.; Muhoberac, B. B.; Dubin, P. L.; Xia, J. Macromolecules 1992, 25, 290.

(24) (a) Rouzina, I.; Bloomfield, V. A. J. Phys. Chem. 1996, 100, 4305; (b) Rouzina, I.; Bloomfield, V. A. J. Phys. Chem. 1996, 110, 4292.

(25) . See, for example, Hunter, R. J. Foundations of Colloid Science; Oxford University Press: Oxford, 1989.

(26) Muthukumar, M. J. Chem. Phys. 1987, 86 (12)

(27) Evers, O. A.; Fleer, G. J.; Scheutjens, J. M. H. M.; Lyklema, J. J. Colloid Interface Sci. 1986, 111, 446.

(28) Wallen, T.; Linse, P. Langmuir 1996, 12, 305.

(29) Odijk, T. Macromolecules 1980, 13, 1542.

(30) von Goeler, F.; Muthukumar, M. J. Chem. Phys. 1994, 100, 7796.

(31) McQuigg, D. W.; Kaplan, J. I.; Dubin, P. L. J. Phys. Chem. 1992, $96,1973$.

(32) Mattison, K. W.; Brittain, I. J.; Dubin, P. L. Biotechnol. Prog. 1995, 11,632 .

(33) Mattison, K. W.; Dubin, P. L.; Brittain, I. J. J. Phys. Chem. 1998, 102, 3830-3836.

(34) (a) Gao, J. Y.; Dubin, P. L.; Muhoberac, B. B. Anal. Chem. 1997 69, 2945. (b) Gao, J. Y.; Dubin, P. L.; Muhoberac, B. B. J. Phys. Chem. 1998, 102, 5529-5535. (c) Gao, J. Y.; Dubin, P. L. Biopolymers, submitted.

(35) Yamanaka, J.; Matsuoka, H.; Kitano, H.; Hasegawa, M.; Ise, N. J. Am. Chem. Soc. 1996, 112 (2), 587.

(36) Practical Handbook of Biochemistry and Molecular Biology; Fasman, G. D., Ed.; CRC Press: Boca Raton, FL, 1989; p 275.

(37) Cannan, R. K.; Palmer, A. H.; Kibrick, A. C. J. Biol. Chem. 1942, 142,803 .

(38) Gao, J. Ph.D. Thesis, Purdue University, 1998. 